\title{
Characterization of Treatments and Disease Course for Women with Breast Cancer Brain Metastases: 5-Year Retrospective Single Institution Experience
}

\author{
Sonya Chew (D) \\ Hailey Kathryn Carroll' \\ Waseem Darwish' \\ Oleksandr Boychak ${ }^{2}$ \\ Michaela Higgins' \\ John McCaffrey' \\ Catherine Margaret Kelly' \\ 'Department of Medical Oncology, Mater \\ Misericordiae University Hospital, \\ Dublin, Ireland; ${ }^{2}$ Department of \\ Radiation Oncology, St Luke's Radiation \\ Oncology Network, Beaumont Hospital, \\ Dublin, Ireland
}

Correspondence: Sonya Chew

Mater Misericordiae University Hospital,

Eccles Street, Dublin 7, Dublin,

D07R2WY, Ireland

Tel +35318032000

Fax +35318032113

Email sonya_chew@yahoo.com
Purpose: Around 30\% of patients with breast cancer will develop brain metastases (BM). We sought to characterize the disease course, treatments and outcome for our patient cohort. Materials and Methods: We extracted clinicopathological data from electronic records from January 2015 to December 2020. Results were generated using SPSS statistics v27.

Results: We identified 98 patients. Median overall survival (OS) from BM diagnosis was 3 months [hormone receptor (HR)+/human epidermal growth factor receptor 2 (HER2)-], 8 months $[\mathrm{HR}+/ \mathrm{HER} 2+], 7$ months [HR-/HER2+] and 2 months [triple negative breast cancer (TNBC)]. Whole brain radiotherapy (WBRT) $(n=48,70 \%)$ was most frequently used followed by surgery $(n=15,22 \%)$ and stereotactic radiosurgery $(n=6,8 \%)$. In patients who received WBRT alone $(n=40)$ the median OS post WBRT was 2.6 months.

Conclusion: After BM development, half of the patients had systemic therapy and $70 \%$ had local therapy, but only the HER2 subgroup had a prolonged OS likely reflecting central nervous system (CNS) activity of anti-HER2 drugs. TNBC patients had the worst prognosis. Although our cohort is small, OS was $>1$ year for $60 \%$ of HER2+ patients who received trastuzumab emtansine after BM development, which is encouraging for antibody drug conjugates and CNS activity. Patients who received WBRT had a higher burden of CNS disease and had an OS of less than 3 months.

Keywords: metastatic, breast cancer, brain metastases, treatment, triple negative

\section{Plain Language Summary}

Around $30 \%$ of patients with breast cancer will develop brain metastases during the course of their disease which can have a devastating effect on prognosis, independence and quality of life. The aim of our retrospective study was to characterize disease course, treatments given and outcome for our patient cohort. We identified 98 patients with metastatic breast cancer and brain metastases. Patients with triple negative breast cancer had the worst prognosis. Overall survival was longest in the HER2-positive subgroup with this cohort receiving the most lines of treatment post brain metastases development. Our results support recent evidence demonstrating central nervous system activity for antibody drug conjugates in this group. Patients who received whole brain radiation had an overall survival of less than 3 months.

\section{Introduction}

Breast cancer (BC) is a disease with a spectrum of distinct biological features that result in differences in effective treatment modalities, response patterns and clinical 
outcomes. Tumours are grouped into surrogate intrinsic subtypes, namely Luminal A, Luminal B, human epidermal growth factor receptor 2 (HER2) positive and basallike. Luminal A tumours are strongly hormone receptor (HR) positive [oestrogen receptor (ER) and progesterone (PR) positive], HER2 negative and have a low proliferative index. Luminal B tumours are ER positive but tend to have a higher proliferative index and varying degrees of PR and HER2 positivity. HER2 positive non-luminal BC are HER2 positive and HR negative while basal-like are triple negative with lack of HR and HER2. ${ }^{1}$

Up to $30 \%$ of patients with $\mathrm{BC}$ will develop brain metastases $(\mathrm{BM})$ during the course of their disease. BM can have a devastating effect on prognosis, independence and quality of life. The incidence of BM and overall survival (OS) differs according to BC subtype. Patients with HER2 overexpression and triple negative breast cancer (TNBC) are at a higher predilection of developing BM. $^{2}$ Treatment options for BC patients with BM can be divided into systemic and local options. The selection of the type of systemic treatment is guided by the BC subtype which include chemotherapy, antibody drug conjugates and HER2 targeting agents. Local therapy options include whole brain radiotherapy (WBRT), stereotactic radiosurgery (SRS) and surgery. We sought to characterize disease course, treatments and outcome for our patient cohort with BM over the last 5 years.

\section{Materials and Methods}

We extracted clinicopathological data using electronic records from January 2015 to December 2020 on BC, HR and HER2 status, time to development of BM, type and number of local and systemic therapies given for $\mathrm{BM}$ and $\mathrm{OS}$ from diagnosis of BM. All BC patients diagnosed with $\mathrm{BM}$ during that time period were included. Both computed tomography (CT) and magnetic resonance imaging (MRI) brain imaging was used for diagnosis of BM and for follow up. Results were generated using SPSS Statistics v27.

\section{Results}

We identified 98 patients. Median age was 49 with a range from 27 to 89 years. Interquartile range (IQR) was 40 to 57 years old. Of the BC subtypes, 39 (40\%) were HR +/HER2-; 44 (45\%) were HER2 positive which were further divided into $29(30 \%) \mathrm{HR}+/ \mathrm{HER} 2+, 15(15 \%)$ HR-/HER2+ and 15 (15\%) triple negative breast cancer (TNBC). The main histology subtype was invasive ductal carcinoma (IDC) at $97 \%$, with the other $3 \%$ consisting of invasive lobular cancer (ILC). Around $60 \%$ of patients had a poorly differentiated tumour (Grade 3 ), with the remaining $40 \%$ being moderately differentiated (Grade 2 ). The median tumour size at initial breast surgery was $40 \mathrm{~mm}$ with a IQR of $22-55 \mathrm{~mm}$. A total $64 \%$ of patients had node positive disease with a median of 1 positive node at initial breast surgery. Of the patients who had node negative disease, $42 \%$ were HER $2+$ and $17 \%$ were TNBC. Despite HR+/HER2- being the most common breast cancer subtype, in our cohort most patients with BM had HER2-positive metastatic breast cancer (MBC). Almost $20 \%$ presented with de novo MBC of which 4 had BM at presentation, and $80 \%$ had relapsed $\mathrm{MBC}$ of which 22 had BM at presentation (Table 1).

At first presentation with MBC (relapsed and de novo) $74 \%$ of patients had no BM, $18 \%$ had BM with extracranial disease and $8 \%$ had $\mathrm{BM}$ only with no extracranial disease (5 had HER2+, 2 had TNBC and 1 had HR +/HER2-). TNBC patients had the highest incidence $(40 \%)$ of presentation of BM at MBC diagnosis. Median time to $\mathrm{BM}$ development from early $\mathrm{BC}$ diagnosis was 69 months (HR+/HER2-), 47 months (HR+/HER2+), 49 months (HR-/HER2+) and 19 months (TNBC). Median time to $\mathrm{BM}$ development from MBC diagnosis was 17 months (HR+/HER2-), 10 months (HR+/HER2+), 12 months (HR-/HER2+) and 3 months (TNBC). A total $22 \%$ of patients developed leptomeningeal disease (LMD) (5 had HER2+, 5 had TNBC and 12 had HR +/HER2-). Three patients developed LMD after BM diagnosis, while 19 presented with concurrent BM and LMD. Median OS was 1.5 months after development of LMD irrespective of tumour subtype.

A total $71 \%$ of patients had systemic treatment prior to developing BM, with a median of 3 lines of treatment (range $1-7)$. Almost half (45\%) of patients had systemic treatment after developing BM with a median of 1 line of treatment. HER2+ patients received the most lines of treatment post BM development with a median of 2 lines of treatment (range $1-6)$. A total $70 \%$ of patients $(n=69)$ received local therapy for $\mathrm{BM}$ with a median of 1 line of treatment (Table 2). WBRT $(n=48,70 \%)$ was the most frequently used modality in the first line followed by surgery $(n=15,23 \%)$ and SRS $(n=6,7 \%)$ (Table 3). Patients with HER2+ BM had the highest incidence of receiving SRS or surgery in the first line (57\%). In patients who received WBRT alone $(n=40)$ the median OS post WBRT was 2.6 months, while in those who received 
Table I Baseline Patient Characteristics and OS

\begin{tabular}{|l|c|c|c|c|c|}
\hline & $\begin{array}{c}\text { All Subtypes } \\
\mathbf{n = 9 8}\end{array}$ & $\begin{array}{c}\text { HR+HER2- } \\
\mathbf{n = 3 9}\end{array}$ & $\begin{array}{c}\text { HR+HER2+ } \\
\mathbf{n = 2 9}\end{array}$ & $\begin{array}{c}\text { HR-HER2+ } \\
\mathbf{n = 1 5}\end{array}$ & $\begin{array}{c}\text { TNBC } \\
\mathbf{n = 1 5}\end{array}$ \\
\hline Median age at breast cancer diagnosis (range) - years & $49(27-89)$ & $48(29-89)$ & $5 I(28-70)$ & $50(27-80)$ & $48(35-75)$ \\
\hline Relapsed MBC - no (\%) & $79(100)$ & $30(38.0)$ & $24(30.4)$ & $12(15.2)$ & $13(16.4)$ \\
\hline De novo MBC - no (\%) & $19(100)$ & $9(47.4)$ & $5(26.3)$ & $3(15.8)$ & $2(10.5)$ \\
\hline $\begin{array}{l}\text { Median time to BM from diagnosis of MBC (range) - } \\
\text { months }\end{array}$ & & $17(0-130)$ & $10(0-78)$ & $12(0-49)$ & $3(0-30)$ \\
\hline Median Breast-GPA score & & 1.0 & 2.5 & 2.5 \\
\hline Median OS from diagnosis of BM (range) - months & & $3(I-47)$ & $8(I-I I 8)$ & $7(I-46)$ & $2(I-26)$ \\
\hline
\end{tabular}

Abbreviations: BM, brain metastases; GPA, Graded Prognostic Assessment; HR, hormone receptor; HER2, human epidermal growth factor receptor 2; MBC, metastatic breast cancer; OS, overall survival; TNBC, triple negative breast cancer.

Table 2 Treatment Received After Diagnosis of BM

\begin{tabular}{|c|c|c|c|c|c|}
\hline & $\begin{array}{l}\text { All Subtypes } \\
\mathrm{n}=69\end{array}$ & $\begin{array}{c}\text { HR+HER2- } \\
n=26\end{array}$ & $\begin{array}{c}\text { HR+HER2+ } \\
n=23\end{array}$ & $\begin{array}{l}\text { HR-HER2+ } \\
\quad n=12\end{array}$ & $\begin{array}{c}\text { TNBC } \\
n=8\end{array}$ \\
\hline $\begin{array}{l}\text { Patients who received systemic treatment after diagnosis of } \\
\text { BM - no (\%) }\end{array}$ & $45(100)$ & $16(35.6)$ & $16(35.6)$ & $9(20.0)$ & $4(8.8)$ \\
\hline $\begin{array}{l}\text { Median no. of systemic treatments after diagnosis of BM } \\
\text { (range) }\end{array}$ & & $2(1-5)$ & $2(I-3)$ & $I(I-6)$ & $I(I-3)$ \\
\hline $\begin{array}{l}\text { Patients who received local treatment after diagnosis of } \\
\text { BM - no (\%) }\end{array}$ & $69(100)$ & $26(37.7)$ & $23(33.3)$ & $12(17.4)$ & $8(11.6)$ \\
\hline Median no. of local treatments after diagnosis of BM (range) & & $I(I-2)$ & $I(I-3)$ & $I(I-2)$ & $I(I-3)$ \\
\hline
\end{tabular}

Abbreviations: BM, brain metastases; HR, hormone receptor; HER2, human epidermal growth factor receptor 2; TNBC, triple negative breast cancer.

Table 3 Types of Local Treatment for BM in the First Line

\begin{tabular}{|l|c|c|c|c|c|}
\hline First Line Local Treatments for BM-no (\%) & $\begin{array}{c}\text { All Subtypes } \\
\mathbf{n = 6 9}\end{array}$ & $\begin{array}{c}\text { HR+HER2- } \\
\mathbf{n = 2 6}\end{array}$ & $\begin{array}{c}\text { HR+HER2+ } \\
\mathbf{n = 2 3}\end{array}$ & $\begin{array}{c}\text { HR-HER2+ } \\
\mathbf{n = 1 2}\end{array}$ & $\begin{array}{c}\text { TNBC } \\
\mathbf{n = 8}\end{array}$ \\
\hline WBRT & $48(69.6)$ & $22(84.7)$ & $16(69.6)$ & $7(58.3)$ & $3(37.5)$ \\
\hline SRS & $6(8.7)$ & $1(3.8)$ & $3(13.0)$ & $0(0)$ & $2(25.0)$ \\
\hline Surgery & $15(21.7)$ & $3(11.5)$ & $4(17.4)$ & $5(49.7)$ & $3(27.5)$ \\
\hline
\end{tabular}

Abbreviations: BM, brain metastases; HR, hormone receptor; HER2, human epidermal growth factor receptor 2; SRS, stereotactic radiosurgery; TNBC, triple negative breast cancer; WBRT, whole brain radiotherapy.

surgery or SRS $(n=21)$ the median time to death was 12 months.

Median OS from BM diagnosis was 3 months (HR +/HER2-), 8 months (HR+/HER2+), 7 months (HR-/ HER2+) and 2 months (TNBC) (Figure 1). The median Breast Graded Prognostic Assessment score (Breast-GPA) was 1.0 (HR+/HER2-), 2.5 (HR+/HER2+), 2.5 (HR-/ HER2+) and 0.5 (TNBC). In the overall cohort, the percentages of patients with a GPA of 0 to $1.0,1.5$ to $2.0,2.5$ to 3.0 and 3.5 to 4.0 were $38 \%, 36 \%, 23 \%$ and $3 \%$ respectively. The median OS was $1,6,10$ and 19 months for a GPA of 0 to $1.0,1.5$ to $2.0,2.5$ to 3.0 and 3.5 to 4.0 respectively (Figure 2). A total $17 \%$ of patients died from extracranial disease progression and their BM were controlled at time of death. A total $34 \%$ died from intracranial progression alone, and $49 \%$ died from progression of both intracranial and extracranial disease. Twelve patients with HER2+ BC who received trastuzumab emtansine (T-DM1) 


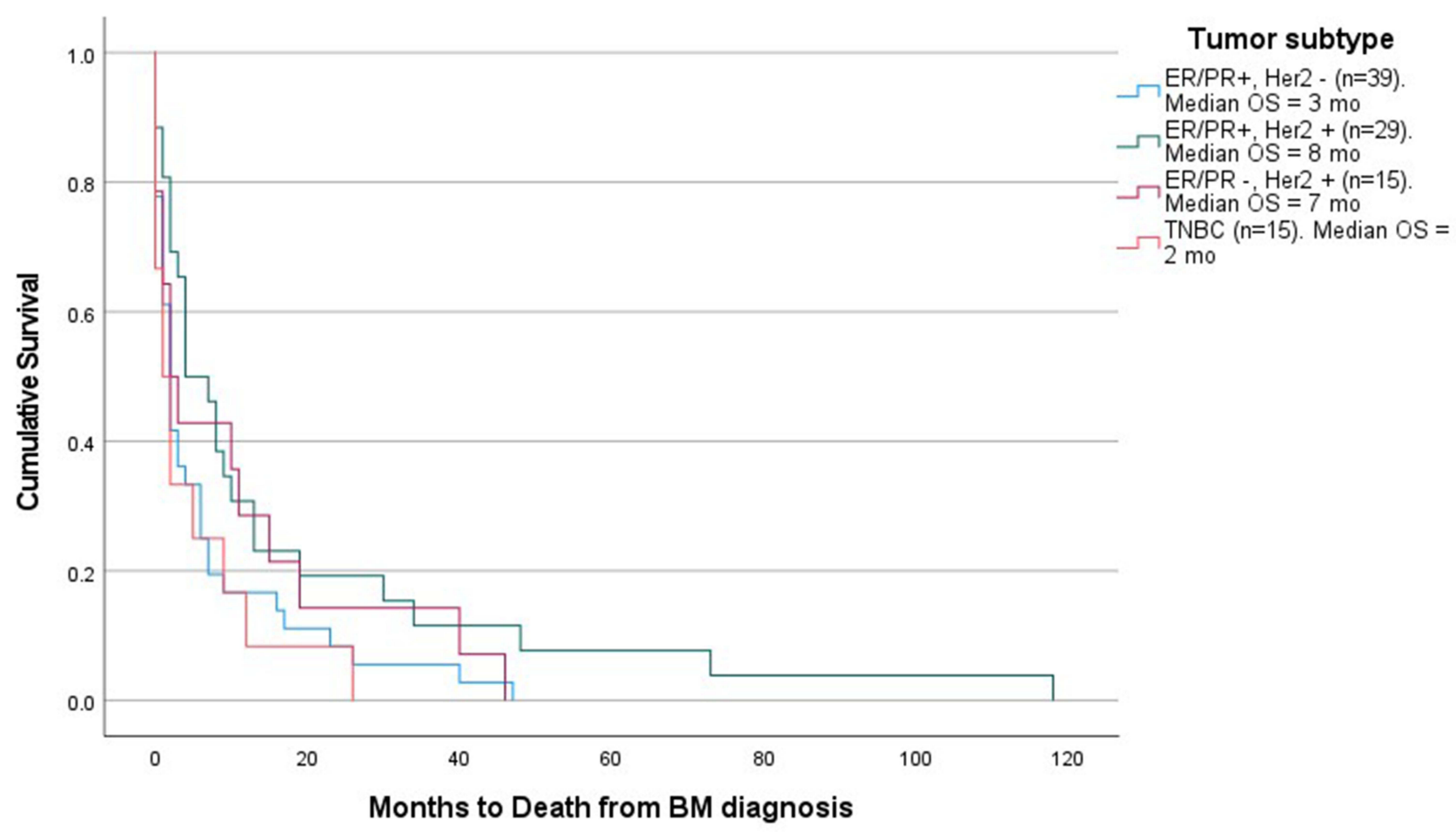

Figure I Kaplan-Meier curve for survival by tumour subtype.

Abbreviations: BM, brain metastases; HR, hormone receptor; HER2, human epidermal growth factor receptor 2; mo, month; OS, overall survival; TNBC, triple negative breast cancer.

prior to $\mathrm{BM}$ appearance had a median time of 10 months to BM development (IQR 6-17 months). For the 13 patients with HER2+ BC given T-DM1 after BM development $60 \%$ had a progression free survival (PFS) of at least 1 year on T-DM1. Nine patients in that cohort proceeded to get subsequent systemic therapies after progressing on T-DM1. Five patients received palbociclib after $\mathrm{BM}$ in the first line and all had progression of disease within 3 months. Considering the OS for the full cohort was so short it is interesting that almost $50 \%$ of patients went on to have further systemic chemotherapy (median 1). This is also noted in the 70\% who received WBRT in the first line of local therapy after which $56 \%$ of that cohort went on to receive further systemic therapy.

\section{Discussion}

$\mathrm{BC}$ is the third most common cancer associated with $\mathrm{BM}$ in the United States after lung cancer and melanoma. ${ }^{3}$ Overall, $10-30 \%$ of patients with MBC will develop BM during the course of their disease which is often the factor that limits survival. ${ }^{4}$ This is seen in our cohort in which over $80 \%$ died from intracranial disease progression. Several risk factors for developing BM have been identified, which include overexpression of HER2, HR negativity, young age at diagnosis and high tumour grade. ${ }^{5}$

The incidence, time to development and prognosis of $\mathrm{BM}$ is influenced by tumour subtype. For the HR +/HER2 - subtype the incidence of BM is $14 \%$ with a median OS of 10 months. $^{6}$ A total $34 \%$ of patients with HER2+ breast cancer will develop BM, however median OS is longer at 17 months (HR-/HER2+) and 22 months (HR+/HER2+). ${ }^{7}$ The incidence of BM is high at $46 \%$ among patients with TNBC with the poorest median OS of 4.9 months. ${ }^{8}$ This is mirrored in a recent study by Kim et al which showed that there was a higher incidence of BM in HER2 and TNBC patients (28.0 and 30.8\%) with a median OS of 12 months (HR+/HER2-), 23 months (HR+/HER2+), 10 months (HR-/HER2+) and 6 months (TNBC) respectively. ${ }^{9}$ The OS for our entire cohort appears shorter than some international data series but this may be due to the relatively small number of patients in our cohort. However, a similar pattern is seen with TNBC patients faring the worst and HER2+ patients having the best prognosis.

The Breast Graded Prognostic Assessment (BreastGPA) is a tool to gauge the prognostic index for patients with BM. The higher the GPA (maximum score 4.0), the 


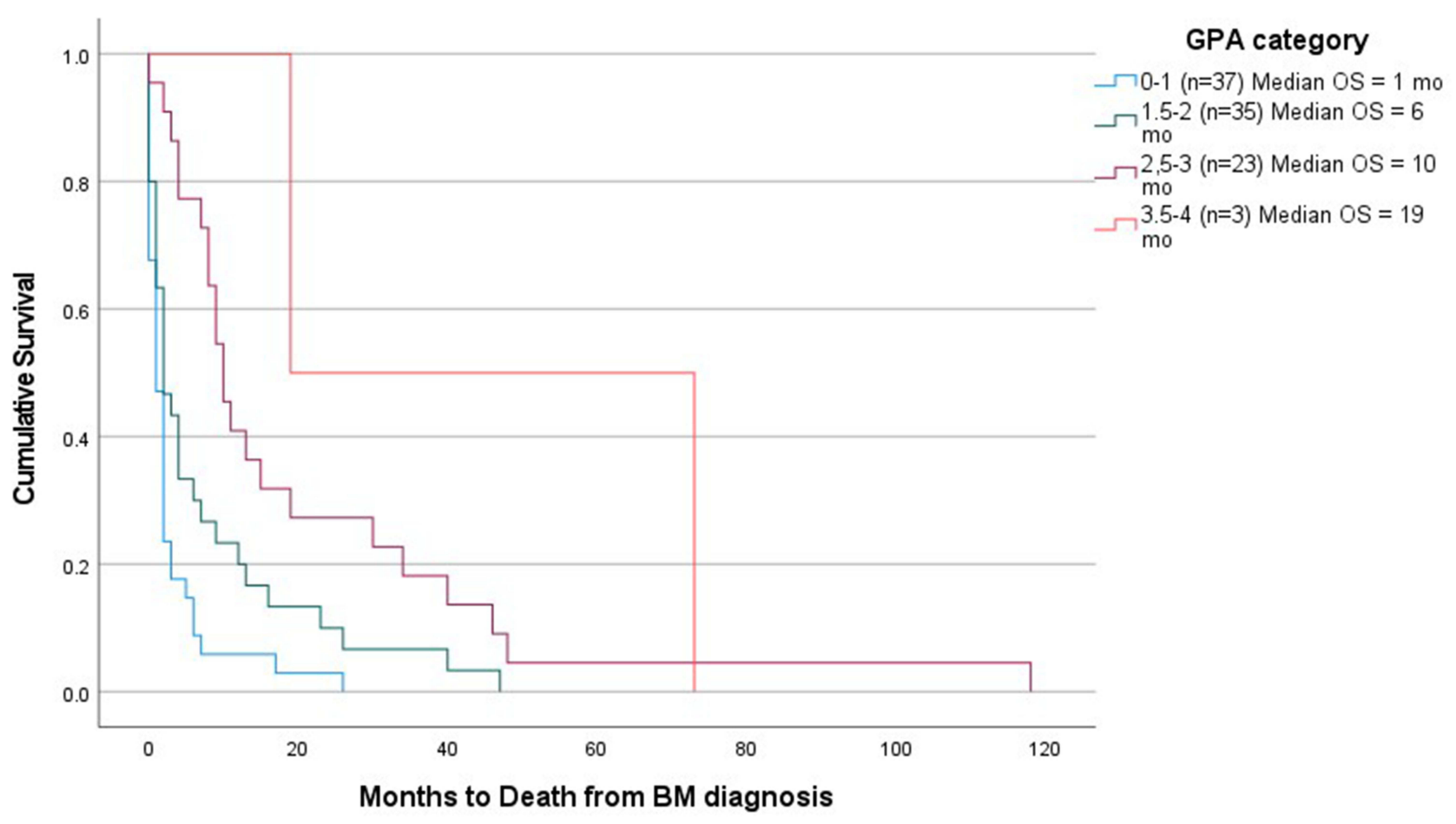

Figure 2 Kaplan-Meier curve for survival by GPA category.

Abbreviations: BM, brain metastases; GPA, Graded Prognostic Assessment; HR, hormone receptor; HER2, human epidermal growth factor receptor 2; mo, month; OS, overall survival; TNBC, triple negative breast cancer.

better the prognosis. For $\mathrm{BC}$, the main prognostic factors are tumour subtype, age, number of BM, presence of extracranial disease and Karnofsky Performance status. Median OS was 6, 13, 24 and 36 months respectively for GPA categories 0 to $1.0,1.5-2.0,2.5-3.0$ and $3.5-4 .^{10}$ This is mirrored in our cohort with patients with higher GPA scores having a better prognosis.

Most of our patients had HR+HER2- disease which makes sense as this is the most common breast cancer subtype. The median time to development of BM for HR +/HER2-, HR+/HER2+, HR-HER2+ and TNBC from initial diagnosis of $\mathrm{BC}$ is 54.4, 47.4, 35.8 and 27.5 months respectively. ${ }^{11}$ TNBC has the highest incidence of $\mathrm{BM}$ at presentation with $\mathrm{MBC}$ which is seen in our cohort (40\%). This is likely due to TNBC having increased risk of presenting with early distant metastases and having the highest risk of developing $\mathrm{BM}$ as the first site of recurrence $(4.7 \%)^{12}$

LMD arises in 11-20\% of diagnosed CNS metastases in $\mathrm{BC}$ and has a median OS of 4.5 to 6 months which does not seem to differ according to tumour subtype. ${ }^{13}$ A similar result was seen in our patient cohort with $22 \%$ patients developing LMD with a median OS of 1.5 months which was similar across all subtypes. None of our patients received intrathecal chemotherapy with literature showing no added survival advantage. ${ }^{14}$

As MBC patients are progressively living longer, they are at risk for additional occurrences of $\mathrm{BM}$ or progression of BM. Over the recent years patients with HER2+ disease have access to multiple new systemic options that have CNS efficacy, but unfortunately in patients with other subtypes like TNBC there has been less progress in treatment options for BM.

Patients with BM have an unmet need as they been traditionally excluded from clinical trials, due to the fact that most of them have a poor prognosis and that many systemic treatments penetrate the blood-brain barrier poorly. ${ }^{15}$ However, with improved treatment efficacy, some patients remain in good clinical condition which has allowed a select MBC cohort with BM to participate in trials.

TNBC patients have limited options of treatment, and the activity of chemotherapy for BM is disappointing with a varied median response rate of about 7 months. ${ }^{16}$ A phase 1 study with capecitabine showed an objective response rate of $18 \%$ with a median response duration of 8 weeks. ${ }^{17}$ Immunotherapy has been a recent exciting new treatment option with improved OS, however these trials 
exclude patients with active BM and thus are unable to comment on the efficacy of immunotherapy on BM. ${ }^{18}$

In patients with the HR+/HER2- subtype, hormonal therapy has poor efficacy with isolated case reports showing modest responses. ${ }^{19,20}$ The focus has shifted to newer targeted therapies, like CDK4/6 and PIK3CA inhibitors. A phase II study with Abemaciclib (a CDK4/6 inhibitor) in heavily pre-treated $\mathrm{MBC}$ patients with active $\mathrm{BM}$ has shown an intracranial clinical benefit rate of $24 \%$ and an intracranial response rate of $5.2 \% .^{21}$ There is an ongoing trial evaluating the response of buparlisib (a PIK3CA inhibitor) with capecitabine in $\mathrm{MBC}$ with $\mathrm{BM}^{22}$

In our cohort patients with BM and HER2+ disease was noted to have a higher likelihood of receiving SRS or brain surgery, more lines of systemic therapy and a better OS. There have been recent breakthroughs in systemic treatments of HER2 $+\mathrm{BC}$ with new agents being able to delay or to stabilize BM. A BM-focused analysis of the CLEOPATRA trial reported a significantly delayed onset of $\mathrm{BM}$ in the pertuzumab arm of 3 months (hazard ratio $=0.58, \mathrm{P}=0.0049) .{ }^{23} \mathrm{~T}-\mathrm{DM} 1$ has shown promising activity in the ongoing KAMILLA trial, with 126 patients with measurable BM showing a best overall response rate and clinical benefit rate of $21.4 \%$ and $42.9 \%$ respectively. Median PFS and OS were 5.5 months and 18.9 months in the 398 patients with baseline BM. ${ }^{24}$ In our HER2+ cohort $30 \%(n=14)$ got T-DM1 after BM development with $60 \%$ of them reaching 1 year of OS while being on T-DM1. New antibody drug conjugates like trastuzumab deruxtecan which has a higher drug-to-antibody ratio than T-DM1 ( 8 versus 3.5 ) and a great bystander antitumour effect due to high membrane permeability of the released payload has shown promising results in phase II trials. ${ }^{25}$ One can hopefully expect an even greater CNS effect and phase III trial results are eagerly awaited. ${ }^{26}$

The HER2CLIMB study is notable for allowing patients with untreated BM to be enrolled. A total 291 patients with $\mathrm{BM}$ in heavily pre-treated HER2+ MBC derived a 4.5 month benefit in OS with tucatinib plus trastuzumab/capecitabine. Among the patients with BM PFS at 1 year was $24.9 \%$ in the tucatinib-combination group and $0 \%$ in the placebocombination group $(\mathrm{P}<0.001){ }^{27}$ Alternative options include lapatinib/capecitabine which was seen in the phase II LANDSCAPE trial to have an objective CNS response in $65 \%$ of enrolled patients. ${ }^{28}$ Neratinib/capecitabine have also shown intracranial activity in a phase II trial with an objective CNS response of $49 \%$ in lapatinib naive patients and $33 \%$ in patients who had previously received lapatinib. ${ }^{29}$
Recent new systemic therapies like tucatinib were not available to our cohort due to lack of reimbursement although it has been approved by the European Medicines Agency (EMA).

Patients with a favourable prognosis of good performance status with controlled or absent extracranial metastasis should be treated aggressively with local therapy. Patients who had surgery for a single BM versus no treatment were found to have improved OS of 40 versus 15 weeks. ${ }^{30}$ WBRT has traditionally been the treatment for unresectable BM. It has the advantages of decreasing the possibility of developing further BM and is more readily available, however is associated with a lack of OS advantage (2.9-4.5 months) and significant neurocognitive toxicity. ${ }^{31} \mathrm{SRS}$ is a preferred treatment option in patients with $\leq 4 \mathrm{BM}$ and has been shown to improve overall survival with a HR of $0.46 .{ }^{32}$ Yamamoto et al found no difference in OS of 10.8 months between patients with 2-4 or 5-10 BM who received SRS. ${ }^{33}$ This could be a tantalizing option for patients who have multiple BM and thus points the way to considering SRS over WBRT even in patients with a greater number of brain metastases.

\section{Conclusion}

Patients with TNBC BM had the shortest prognosis with poor OS and those with HER2-positive BM had the best prognosis and received more systemic and local therapies after BM diagnosis. Recent drugs such as trastuzumab deruxtecan have shown promising activity and should improve outcome for our patients in the future. Although our cohort is small, OS was $>1$ year for $60 \%$ of HER2+ patients who received T-DM1 after BM development which is encouraging for antibody drug conjugates and CNS activity. Overall survival was poor at less than 3 months for patients who received WBRT indicating these patients had a higher burden of disease in the CNS.

\section{Institutional Review Board Statement}

The study was conducted according to the guidelines of the Declaration of Helsinki. Ethical review and approval were waived for this study, as this was a retrospective observational study of data obtained for clinical purposes. This study was registered under CA 20-061 and approved by the Clinical Audit, Quality \& Patient Safety Directorate, Mater Misericordiae University Hospital, Dublin, Ireland on 29 October 2020. 


\section{Data Sharing Statement}

The data presented in this study are available on request from the corresponding author. The data are not publicly available due to privacy reasons and GDPR.

\section{Informed Consent Statement}

Patient consent was waived due to retrospective data being collected anonymously for a quality improvement process that aimed to improve patient care and outcomes through systematic review of care against explicit criteria and standards.

\section{Acknowledgments}

The abstract of this paper was presented at the 2021 ASCO Annual Meeting as a virtual presentation with interim findings. The abstract was published in "Virtual Abstracts" in Journal of Clinical Oncology 2021 39:15_suppl, e13055e13055. DOI: 10.1200/JCO.2021.39.15_suppl. “e13055”

\section{Funding}

This research received no external funding.

\section{Disclosure}

The authors declare no conflicts of interest.

\section{References}

1. Cardoso F, Kyriakides S, Ohno S, et al. Early breast cancer: ESMO Clinical Practice Guidelines for diagnosis, treatment and follow-up. Ann Oncol. 2019;30(8):1194-1220.

2. Niwińska A, Murawska M, Pogoda K. Breast cancer brain metastases: differences in survival depending on biological subtype, RPA RTOG prognostic class and systemic treatment after whole-brain radiotherapy (WBRT). Ann Oncol. 2010;21(5):942-948. doi:10.1093/annonc/mdp 407

3. Barnholtz-Sloan JS, Sloan AE, Davis FG, Vigneau FD, Lai P, Sawaya RE. Incidence proportions of brain metastases in patients diagnosed (1973 to 2001) in the Metropolitan Detroit Cancer Surveillance System. J Clin Oncol. 2004;22(14):2865-2872. doi:10. 1200/JCO.2004.12.149

4. Witzel I, Oliveira-Ferrer L, Pantel K, Müller V, Wikman H. Breast cancer brain metastases: biology and new clinical perspectives. Breast Cancer Res. 2016;18(1):8. doi:10.1186/s13058-015-0665-1

5. Tham YL, Sexton K, Kramer R, Hilsenbeck S, Elledge R. Primary breast cancer phenotypes associated with propensity for central nervous system metastases. Cancer. 2006;107(4):696-704. doi:10.1002/ cncr.22041

6. Brosnan EM, Anders CK. Understanding patterns of brain metastasis in breast cancer and designing rational therapeutic strategies. Ann Transl Med. 2018;6(9):163. doi:10.21037/atm.2018.04.35

7. Bendell JC, Domchek SM, Burstein HJ, et al. Central nervous system metastases in women who receive trastuzumab-based therapy for metastatic breast carcinoma. Cancer. 2003;97(12):2972-2977. doi:10. $1002 /$ cncr. 11436

8. Lin NU, Claus E, Sohl J, Razzak AR, Arnaout A, Winer EP. Sites of distant recurrence and clinical outcomes in patients with metastatic triple-negative breast cancer: high incidence of central nervous system metastases. Cancer. 2008;113(10):2638-2645. doi:10.1002/cncr.23930
9. Kim YJ, Kim JS, Kim IA. Molecular subtype predicts incidence and prognosis of brain metastasis from breast cancer in SEER database. J Cancer Res Clin Oncol. 2018;144(9):1803-1816. doi:10.1007/ s00432-018-2697-2

10. Sperduto PW, Mesko S, Li J, et al. Beyond an updated graded prognostic assessment (Breast GPA): a prognostic index and trends in treatment and survival in breast cancer brain metastases from 1985 to today [published correction appears in Int $\mathrm{J}$ Radiat Oncol Biol Phys. 2021 Jan 1;109(1):303]. Int J Radiat Oncol Biol Phys. 2020;107(2):334-343. doi:10.1016/j.ijrobp.2020.01.051

11. Sperduto PW, Kased N, Roberge D, et al. The effect of tumor subtype on the time from primary diagnosis to development of brain metastases and survival in patients with breast cancer. $J$ Neurooncol. 2013;112(3):467-472. doi:10.1007/s11060-013-1083-9

12. Jin J, Gao Y, Zhang J, et al. Incidence, pattern and prognosis of brain metastases in patients with metastatic triple negative breast cancer. BMC Cancer. 2018;18(1):446. doi:10.1186/s12885-018-4371-0

13. Lee S, Ahn HK, Park YH, et al. Leptomeningeal metastases from breast cancer: intrinsic subtypes may affect unique clinical manifestations. Breast Cancer Res Treat. 2011;129(3):809-817. doi:10.1007/s10549-011-1682-0

14. Lee YC, Hsieh CC, Chuang JP, Li CY. The necessity of intrathecal chemotherapy for the treatment of breast cancer patients with leptomeningeal metastasis: a systematic review and pooled analysis. Curr Probl Cancer. 2017;41(5):355-370. doi:10.1016/j.currproblcancer.20 17.07.001

15. Gounder MM, Spriggs DR. Inclusion of patients with brain metastases in Phase I trials: an unmet need. Clin Cancer Res. 2011;17 (12):3855-3857. doi:10.1158/1078-0432.CCR-11-0759

16. Rosner D, Nemoto T, Lane WW. Chemotherapy induces regression of brain metastases in breast carcinoma. Cancer. 1986;58(4):832-839. doi:10.1002/1097-0142(19860815)58:4<832::aidcncr2820580404>3.0.co;2-w

17. Rivera E, Meyers C, Groves M, et al. Phase I study of capecitabine in combination with temozolomide in the treatment of patients with brain metastases from breast carcinoma. Cancer. 2006;107 (6):1348-1354. doi:10.1002/cncr.22127

18. Kim JS, Kim IA. Evolving treatment strategies of brain metastases from breast cancer: current status and future direction. Ther $A d v$ Med Oncol. 2020;12:1758835920936117. doi:10.1177/175883592 0936117

19. Salvati M, Cervoni L, Innocenzi G, Bardella L. Prolonged stabilization of multiple and single brain metastases from breast cancer with tamoxifen. Report of three cases. Tumori J. 1993;79(5):359-362. doi:10.1177/030089169307900516

20. Wang Q, Sun B, Liu C, et al. Brain metastases from breast cancer may respond to endocrine therapy: report of two cases. Onco Targets Ther. 2019;12:1389-1393. doi:10.2147/OTT.S188143

21. Tolaney SM, Sahebjam S, Le Rhun E, et al. A phase II study of abemaciclib in patients with brain metastases secondary to hormone receptor-positive breast cancer. Clin Cancer Res. 2020;26 (20):5310-5319. doi:10.1158/1078-0432.CCR-20-1764

22. Phase II multicenter single-arm study of BKM120 plus capecitabine for breast cancer patients with brain metastases. Available from: https://clinicaltrials.gov/ct2/show/NCT02000882. Accessed July 26, 2021.

23. Swain SM, Baselga J, Miles D, et al. Incidence of central nervous system metastases in patients with HER2-positive metastatic breast cancer treated with pertuzumab, trastuzumab, and docetaxel: results from the randomized phase III study CLEOPATRA. Ann Oncol. 2014;25(6):1116-1121. doi:10.1093/annonc/mdu133

24. Montemurro F, Delaloge S, Barrios CH, et al. Trastuzumab emtansine (T-DM1) in patients with HER2-positive metastatic breast cancer and brain metastases: exploratory final analysis of cohort 1 from KAMILLA, a single-arm phase IIIb clinical trial. Ann Oncol. 2020;31(10):1350-1358. doi:10.1016/j.annonc.2020.06.020 
25. Modi S, Saura C, Yamashita T, et al. Trastuzumab deruxtecan in previously treated HER2-positive breast cancer. $N$ Engl J Med. 2020;382(7):610-621. doi:10.1056/NEJMoa191 4510

26. A phase 3, multicenter, randomized, open-label, active-controlled study of trastuzumab deruxtecan (DS-8201a), an anti-HER2antibody drug conjugate, versus treatment of investigator's choice for HER2-positive, unresectable and/or metastatic breast cancer subjects previously treated with T-DM1. Available from: https://clinicaltrials.gov/ct2/show/NCT03523585. Accessed July 26, 2021.

27. Murthy RK, Loi S, Okines A, et al. Tucatinib, trastuzumab, and capecitabine for HER2-positive metastatic breast cancer. N Engl J Med. 2020;382(7):597-609. doi:10.1056/NEJMoa1914609

28. Bachelot T, Romieu G, Campone M, et al. Lapatinib plus capecitabine in patients with previously untreated brain metastases from HER2-positive metastatic breast cancer (LANDSCAPE): a single-group Phase 2 study. Lancet Oncol. 2013;14(1):64-71. doi:10.1016/S1470-2045(12)70432-1
29. Freedman RA, Gelman RS, Anders CK, et al. TBCRC 022: a phase II trial of neratinib and capecitabine for patients with human epidermal growth factor receptor 2-positive breast cancer and brain metastases. J Clin Oncol. 2019;37(13):1081-1089. doi:10.1200/JCO.18.01511

30. Patchell RA, Tibbs PA, Walsh JW, et al. A randomized trial of surgery in the treatment of single metastases to the brain. $N$ Engl J Med. 1990;322(8):494-500. doi:10.1056/NEJM199002223220802

31. Jeene PM, de Vries KC, van Nes JGH, et al. Survival after whole brain radiotherapy for brain metastases from lung cancer and breast cancer is poor in 6325 Dutch patients treated between 2000 and 2014. Acta Oncol. 2018;57(5):637-643. doi:10.1080/0284186X.2017.1418534

32. Halasz LM, Uno H, Hughes M, et al. Comparative effectiveness of stereotactic radiosurgery versus whole-brain radiation therapy for patients with brain metastases from breast or non-small cell lung cancer. Cancer. 2016;122(13):2091-2100. doi:10.1002/cncr.30009

33. Yamamoto M, Serizawa T, Shuto T, et al. Stereotactic radiosurgery for patients with multiple brain metastases (JLGK0901): a multi-institutional prospective observational study. Lancet Oncol. 2014;15(4):387-395. doi:10.1016/S1470-2045(14)70061-0

\section{Publish your work in this journal}

Cancer Management and Research is an international, peer-reviewed open access journal focusing on cancer research and the optimal use of preventative and integrated treatment interventions to achieve improved outcomes, enhanced survival and quality of life for the cancer patient.
The manuscript management system is completely online and includes a very quick and fair peer-review system, which is all easy to use. Visit http://www.dovepress.com/testimonials.php to read real quotes from published authors. 